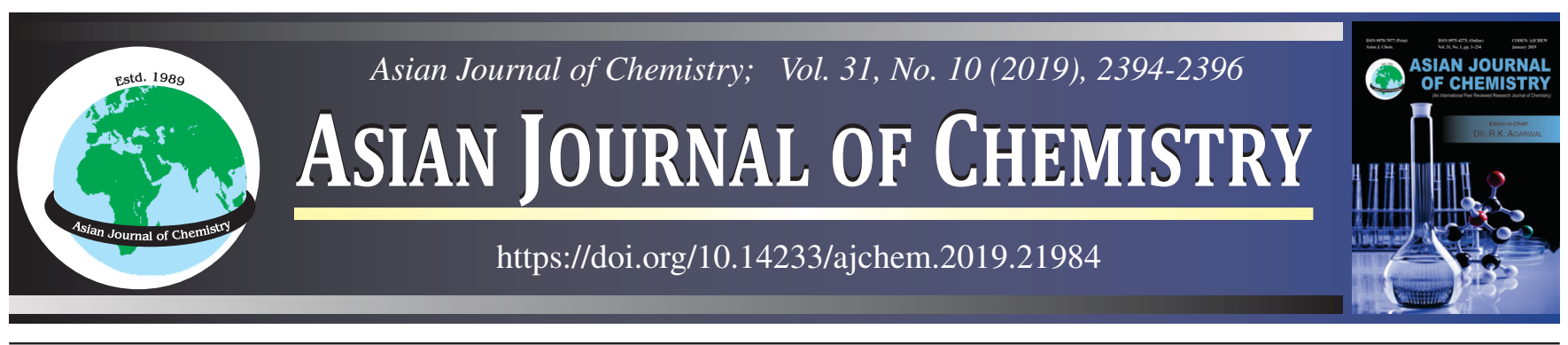

\title{
Synthesis of Eco-Friendly Detergent Based on Crude Palm Oil and Titania Nanoparticles
}

P.P. Wulandari, M.T. Adiwibowo, A.S. Redjeki, M. Ibadurrohman and Slamet*

Department of Chemical Engineering, Universitas Indonesia, Kampus UI Depok, Depok, Indonesia

*Corresponding author: Tel/Fax: +62 21 7863516; E-mail: slamet@che.ui.ac.id

Received: 7 February 2019;

Accepted: 17 July 2019;

Published online: 30 August 2019;

AJC-19558

| In this work, we report the synthesis of eco-friendly detergent from crude palm oil and titania. Crude palm oil was converted into methyl ester sulfonate through esterification, transesterification and followed by sulfonation process. As-produced methyl ester sulfonate was characterized with Fourier transformed infrared spectroscopy (FTIR), gas chromatography-mass spectroscopy (GC-MS), liquid chromatography mass spectroscopy (LCMS) and surface tension analysis. Detergents were synthesized by dispersing titania nanoparticles into methyl ester sulfonate solution, forming a nanofluid. Stability and detergency of the nanofluidic detergents were then evaluated. The results showed that the nanofluid detergents were stable in the presence of $0.1 \mathrm{wt} \%$ titania and the photocatalytic activity of titania nanoparticles improved the ability of nanofluidic detergents to remove dirt as well as degrading the surfactant residues in the laundry waste stream.

Keywords: Cleaning agent, Eco-friendly detergent, Methyl ester sulfonate, Nanofluid, Titania.

\section{INTRODUCTION}

Surfactant is a surface-active agent that is commonly present in commercial detergent or other personal care and household products [1]. It has an amphiphilic structure possessing both polar water-soluble and non-polar water-insoluble components (usually a long hydrocarbon chain). Due to its amphiphilic structure, surfactant is used as a cleaning agent to remove dirt and stains. Based on its hydrophilic group, surfactants are classified as anionic, nonionic, cationic and amphoteric. As a key component in those products, surfactant plays a crucial role in reducing the surface tension of fluids.

Up to this day, industrial production of detergents is predominantly based on anionic petrochemical-based surfactants, such as linear alkyl benzene sulfonate (LABS). In recent years, however, the use of linear alkyl benzene sulfonate has increased major concern with respect to its detrimental impacts on aquatic ecosystems. Enormous efforts have been exerted in order to mitigate the environmental impacts of detergents. It is suggested that methyl esther sulfonate (MES) is one of the most promising candidates which could substitute linear alkyl benzene sulfonate for detergent application. Importantly, methyl ester sulfonate can be produced from bio-based oil such as crude palm oil, soybean oil, curcas oil, tallow, among others. As the world's largest producer of crude palm oil, Indonesia has a huge potential to produce eco-friendly detergents based on methyl ester sulfonate. Utilizing crude palm oil to produce detergent is expected to increase the economic value of crude palm oil, which is currently process for food industry only.

Nanofluid is a term used to describe a suspension of nanoparticles (size particle smaller than $100 \mathrm{~nm}$ ) such as $\mathrm{TiO}_{2}, \mathrm{ZnO}$, $\mathrm{Fe}_{3} \mathrm{O}_{4}$ and $\mathrm{Cu}$, which are dispersed in host liquids such as water, ethylene glycol, engine oil and others [2-5]. Nanofluid has a wide scope of applications in many fields, such as antibacterial agent and photocatalysis [6]. In the context of photocatalysis, $\mathrm{TiO}_{2}$ is the most widely used materials in many photocatalytic applications due to its excellent photo-activity and stability, vast availability, cost-effectiveness and non-toxicity. The photocatalytic activity of titania has been used by researchers for various pollutant degradation such as pesticide degradation [7], organic pollutant [8], E. coli elimination from wastewater [9] and ammonia [10].

Considering these virtues, combination of methyl ester sulfonate and titania in the form of a nanofluidic suspension as household detergents are expected to give many benefits. The presence of titania photocatalysts would facilitate an in

This is an open access journal, and articles are distributed under the terms of the Attribution 4.0 International (CC BY 4.0) License. This license lets others distribute, remix, tweak, and build upon your work, even commercially, as long as they credit the author for the original creation. You must give appropriate credit, provide a link to the license, and indicate if changes were made. 
situ degradation of dirt in the wastewater stream, as well as the residual surfactant itself. The stability of titania nanoparticles in the nanofluidic system could also be improved by adjusting the $\mathrm{pH}$ to a slightly acidic condition (based on the zeta potential of titania). Lime can be added into the nanofluid system, not only as titania stabilizer but also because it is also commonly used as a cleaning agent for utensils. In this paper, we report the synthesis of crude palm oil-based surfactant and synthesis of nanofluidic detergents from crude palm oil-based surfactant and titania nanoparticles as an eco-friendly cleaning agent. We also investigated the stability and detergency of the nanofluid detergents.

\section{EXPERIMENTAL}

Crude palm oil (CPO) was purchased from a local plantation in Lampung, Indonesia, while methanol, $\mathrm{H}_{2} \mathrm{O}_{2}, \mathrm{KOH}, \mathrm{NaOH}$, methylene blue and chloroform were purchased from Merck. Sulfuric acid is purchased from Mallinckrodt, $\mathrm{NaHSO}_{3}$ from PT Smartlab Indonesia and $\mathrm{TiO}_{2}$-P25 (79:21 anatase: rutile) was purchased from Evonik. Aquadest as a solvent of surfactant and host fluid for titania was purchased from Wiloso Chemical. All chemicals are of analytical grade and used as received without further treatment. Lime and technical grade of carboxy methyl cellulose (CMC) were purchased from a local market in Jakarta and a local chemical agent in Jakarta, respectively.

The synthesis of methyl ester sulfonate was conducted via three main processes, namely: esterification, transesterification and sulfonation, which have been reported elsewhere [11]. From our previous study, we obtained that the optimum condition for esterification process was at $60{ }^{\circ} \mathrm{C}$ with $1.5 \mathrm{~h}$ reaction, in the presence of $1 \% \mathrm{v} / \mathrm{v} \mathrm{H}_{2} \mathrm{SO}_{4}$ catalyst and crude palm oil-methanol molar ratio of 1:6. Transesterification was performed by heating the fatty acid ester and triglyceride at $60{ }^{\circ} \mathrm{C}$ for $1 \mathrm{~h}$ with $1 \% \mathrm{w} / \mathrm{v} \mathrm{KOH}$ catalyst and crude palm oilmethanol molar ratio of 1:6. Glycerol (a by-product of both processes) were separated by a precipitation method. The asproduced methyl ester was then washed with deionized water at $65{ }^{\circ} \mathrm{C}$ 3-4 times in order to remove glycerol and soap that are formed during reactions. The methyl ester was dried by heating and stirring until water bubbles disappeared on the surface. Methyl ester sulfonate was produced by sulfonating methyl ester with $\mathrm{NaHSO}_{3}$ in $4.5 \mathrm{~h}$ at $100{ }^{\circ} \mathrm{C}$, with methyl ester- $\mathrm{NaHSO}_{3}$ molar ratio of $1: 1.5$. The synthesized methyl ester and methyl ester sulfonate were characterized by GCMS and FTIR spectrophotometer in the mid-IR region, ranging from 4000 to $400 \mathrm{~cm}^{-1}$. An amount of $0.1 \% \mathrm{w}$ of titania nanoparticles was then added into the resultant methyl ester sulfonate produced along with lime and CMC. Detergency of the produced surfactant was assessed in the following fashion. A piece of cloth was dipped in a methylene blue solution (2 ppm) for $4 \mathrm{~h}$. The stained cloth was washed in a photoreactor-washing machine with/without mercury lamp. The concentration of methylene blue was monitored by measuring the absorbance of the solution (using UV-vis spectrometer) before and after the washing process. The ability of titania to degrade surfactant residues was measured with methylene blue active substances (MBAS) method. The stability of the nanofluid is measured by comparing the absorbance at $652 \mathrm{~nm}$ every hour for $3 \mathrm{~h}$.

\section{RESULTS AND DISCUSSION}

Methyl ester sulfonate was produced from crude palm oil through esterification/transesterification reaction, continued with sulfonation, purification and neutralization. From previous study, the optimum methanol:FFA mole ratio is $6: 1$ with conversion reaching $95.52 \%$, while the optimum mole ratio of methanol:triglyceride is also 6:1 with conversion of $96.0 \%$ [11].

Fig. 1 shows the FTIR spectra of crude palm oil (a), FAME (b) and methyl ester sulfonate (c). Strong peaks at 2917 and $2850 \mathrm{~cm}^{-1}$ are typical for C-H alkane group while a strong signal at $1744 \mathrm{~cm}^{-1}$ corresponds to $\mathrm{C}=\mathrm{O}$ vibration. The presence of methyl group $\left(-\mathrm{CH}_{3}\right)$ is confirmed by the peak at $1462 \mathrm{~cm}^{-1}$, while the $\mathrm{C}-\mathrm{O}$ group entails the appearance of a peak situated at $1159.90 \mathrm{~cm}^{-1}$. In the case of sulfonated methyl ester (Fig. 1c), the peak at $1216 \mathrm{~cm}^{-1}$ implies the presence of sulfonate group as it was suggested that the stretching vibration of $\mathrm{C}=\mathrm{O}$ sulfonate group is indicated by the signals in the wavenumber interval of $1250-1050 \mathrm{~cm}^{-1}$ [12]. Methyl ester sulfonate produced was then used for the detergent formulation, into which lime and titania nanoparticles were added.

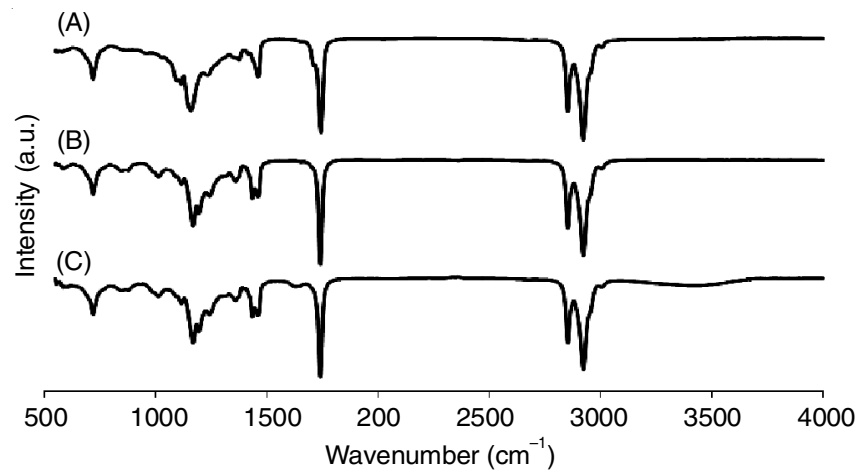

Fig. 1. FTIR analysis of (A) crude palm oil, (B) fatty acid methyl ester and (C) methyl ester sulfonate

In a nanofluidic system, stability is a major concern. The van der Waals force between nanoparticles tend to create bonds and electrostatic attraction which leads to eventual agglomeration and precipitation. Surfactant addition is one of the common methods used to maintain nanofluid stability. In the presence of surfactant, nanofluid will modify hydrophobic groups of nanoparticles into hydrophilic in aqueous liquid media and vice-versa in non-aqueous liquid media. Besides the evolution of hydrophobic-hydrophilic behaviour, the repulsion force between nanoparticles in the suspension system becomes stronger due to the increase of surface charges. Fig. 2 clearly shows the significance of surfactant in maintaining the stability of nanofluidic detergent. In the presence of methyl ester sulfonate (an anionic surfactant), titania particles surface will adsorb the hydrophobic tail and it becomes negatively charged. The fact that these particles possess the same type of charge gives rise to repulsion force between them, hence hindering agglomeration. Fig. 2 showed that the stability of nanofluids increases with increasing surfactant concentration. The presence of a sufficient amount of surfactant is necessary to ensure that the repulsion force between particles would compromise the van der Waals force. Stability of nanofluid system 


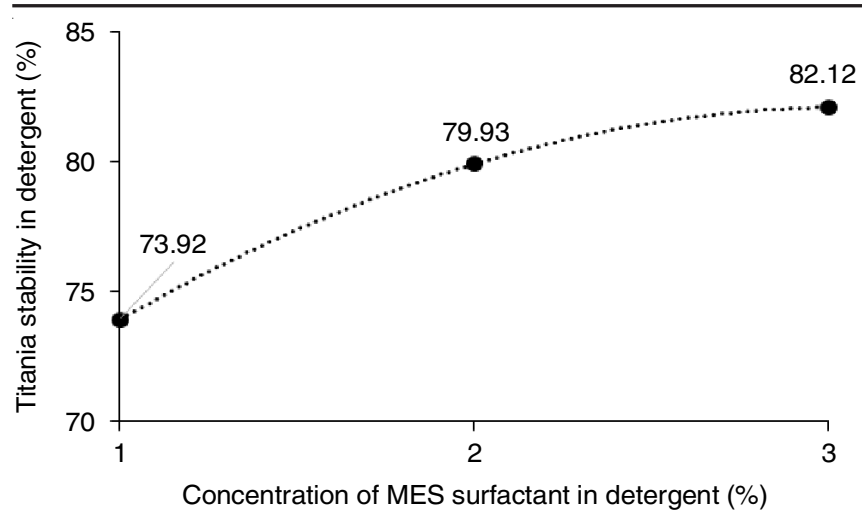

Fig. 2. Stability of nanofluid in the presence of surfactant

is also affected by $\mathrm{pH}$ of the suspension. In this study, $\mathrm{pH}$ adjustment of the nanofluid was performed by adding lime to the system. Addition of $2 \mathrm{~mL}$ lime into the system, set the $\mathrm{pH}$ value to 4 . In acidic media, titania nanoparticles would adsorb liquids, inducing positive charge on the surface [13]. An anionic surfactant like methyl ester sulfonate, which is negatively charged in its hydrophilic head tend to move away from titania nanoparticles due to electrostatic repulsion force.

The ability of the detergent to remove dirt is assessed with methylene blue as a dirt (stain) model, of which results are depicted in Fig. 3. It is revealed that $3 \%(w / w)$ of methyl ester sulfonate in the detergent formulation could remove methylene blue up to $74 \%$ in the case of no illumination and $85 \%$ in the case of illumination by mercury lamp.

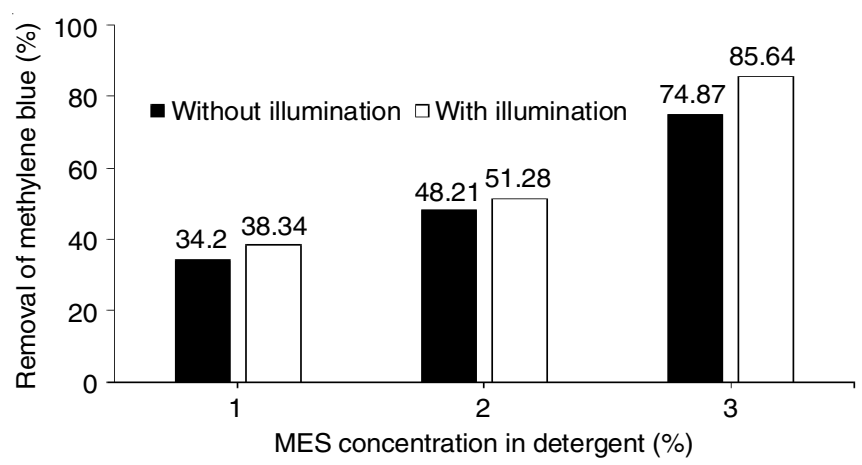

Fig. 3. Detergency test for various methyl ester sulfonate concentration

Fig. 4 presents the results of degradation test of surfactant residues in laundry wastewater. Degradation of surfactant in wastewater increase with surfactant concentration in detergent. In the presence of $3 \%$ surfactant in detergent, the final content of surfactant residues is around $40 \%$ without illumination and around $33 \%$ when exposed to illumination. In the case where the nanofluidic detergent is exposed to photons, titania produced positively charged holes, along with electrons. These excitons are responsible for the elimination of residual surfactants that are present in the wastewater stream.

\section{Conclusion}

In this paper, we reported the synthesis and application of nanofluidic detergent comprising of crude palm oil-based methyl ester sulfonate (MES) and titania nanoparticles. The use of bio-based surfactant in detergent products ensures more eco-friendly household activity. The stability of titania nanofluid is enhanced by the presence of surfactant which modifies

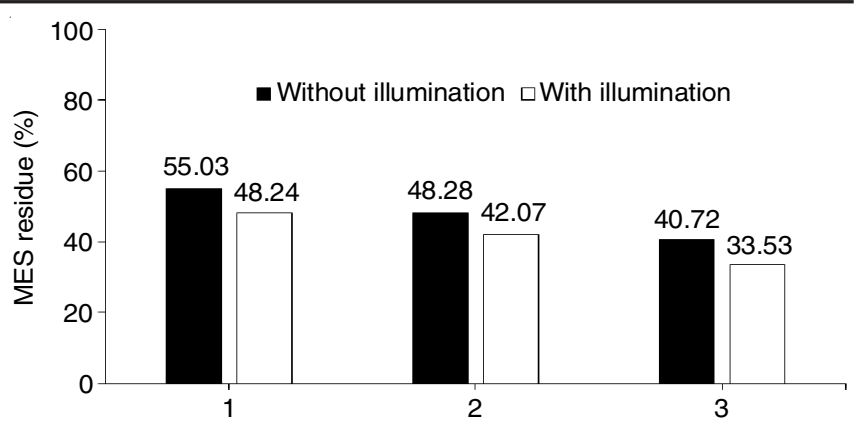

MES concentration in detergent (\%)

Fig. 4. Residue of methyl ester sulfonate in various methyl ester sulfonate concentration

the surface charge of titania nanoparticles hence inducing electrostatic repulsion. This repulsion force prevents these particles from undesired agglomeration. In terms of detergency, methyl ester sulfonate-based detergent shows good removal of dirt (modeled by methylene blue). In the presence of titania nanoparticles, methylene blue is not only collected by methyl ester sulfonate, but it is also irreversibly eliminated by photocatalytic activity of titania.

\section{ACKNOWLEDGEMENTS}

The authors acknowledge financial support from Ministry of Research, Technology and Higher Education of the Republic of Indonesia (RISTEK DIKTI) through INSINAS 2018 Program-Contract number: 253/UN2.R3.1/HKP.05.00/2018.

\section{CONFLICT OF INTEREST}

The authors declare that there is no conflict of interests regarding the publication of this article.

\section{REFERENCES}

1. L.H.T. Tai and V. Nardello-Rataj, OCL-OL Corps Gras Lipid., 8, 141 (2001).

2. M.T. Adiwibowo, M. Ibadurrohman and Slamet, Int. J. Technol., 9, 307 (2018);

https://doi.org/10.14716/ijtech.v9i2.1065.

3. Slamet and A.S. Redjeki, AIP Conf. Proceed., 1904, 020063 (2017); https://doi.org/10.1063/1.5011920.

4. M. Sheikholeslami and K. Vajravelu, J. Mol. Liq., 233, 203 (2017); https://doi.org/10.1016/j.molliq.2017.03.026.

5. S. Aberoumand and A. Jafarimoghaddam, J. Taiwan Inst. Chem. Eng., 71, 315 (2017);

https://doi.org/10.1016/j.jtice.2016.12.035.

6. L. Zhang, Y. Ding, M. Povey and D. York, Prog. Nat. Sci., 18, 939 (2008); https://doi.org/10.1016/j.pnsc.2008.01.026.

7. M. Cruz, C. Gomez, C.J. Duran-Valle, L.M. Pastrana-Martínez, J.L. Faria, A.M. Silva, M. Faraldos and A. Bahamonde, Appl. Surf. Sci., 416, 1013 (2017);

https://doi.org/10.1016/j.apsusc.2015.09.268.

8. M. Yan, G. Zeng, X. Li, C. Zhao, G. Yang, J. Gong, G. Chen, L. Tang and D. Huang, New J. Chem., 41, 4377 (2017); https://doi.org/10.1039/C6NJ03196J.

9. J. Murcia, E. Ávila-Martínez, H. Rojas, J. Navío and M. Hidalgo, Appl. Catal. B, 200, 469 (2017);

https://doi.org/10.1016/j.apcatb.2016.07.045.

10. Y.-N. Li, Z.-Y. Chen, S.-J. Bao, M.-Q. Wang, C.-L. Song, S. Pu and D. Long, Chem. Eng. J., 331, 383 (2018); https://doi.org/10.1016/j.cej.2017.08.119.

11. Slamet, M. Ibadurrohman and P.P. Wulandari, AIP Conf. Proceed., 1904, 020058 (2017);

https://doi.org/10.1063/1.5011915.

12. R.M. Silverstein, F.X. Webster, D.J. Kiemle and D.L. Bryce, Spectrometric Identification of Organic Compounds, John Wiley \& Sons (2014).

13. G. Wang, Y. Morikawa, T. Matsumoto and J. Nakamura, J. Phys. Chem. $B, \mathbf{1 1 0}, 9$ (2006); https://doi.org/10.1021/jp055689e. 